\title{
Influencing factors on changes in nutritional behaviour over the life span
}

\section{Abstract}

From an early age nutrition is part of everyday life, but the motives of eating change over the life span. The nutrition depends on many factors and is always embedded in an individual and social dimension. Especially life-phase-specific framework conditions and factors play an important role in the daily nutrition. The aim of this project was to find out how different factors affect nutritional behaviour in different age groups.

The study was part of the research project "enable - healthy food choices in all stages of life". In order to examine the factors influencing the nutritional behaviour, focus groups with young women and men, middle-agers and elderly had been implemented. In addition biographical interviews were conducted to gain a deeper insight into the reasons for changing the nutritional behaviour. Thus influencing factors and their weighting could be identified and their mode of action could be linked.

For example, changes in the nutritional habits of adolescents could be figured out more accurately. Essential for young adults is the replacement of the parents, which is obtained by moving out of the parental home. An own lifestyle and nutritional style has to be developed and there are many changes and new freedoms and opportunities to try out. The consumption of fast food, for example, is particularly attractive to adolescents, as it is a distinction from the adult culture and rules of eating, like eating with cutlery. Another example is the nutritional situation of elderly, which is often characterized by malnutrition or disease-related dietary requirements. However, many elderly do not eat as recommended, even if they have the skills to implement it. In everyday life, deviations from the nutritional recommendations are often legitimized by the age itself "now I can indulge myself a bit". Other legitimization factors include the death of the partner "I have been cooking my whole life, now I can just eat a sandwich".

The majority of people do not eat according to nutritional recommendations, too much sugar and salt and little fruits and vegetables characterize the nutrition. The consequences do not only concern health aspects such as nutritional diseases, but also ecological aspects. Despite knowing about the consequences of unhealthy food, many do not realize their knowledge about healthy eating in everyday life. If the nutritional situation should be improved sustainably, it is therefore necessary to include the individual factors and changes in the life span in considerations and concepts of nutritional programs.

\section{Conflict of Interest}

There is no conflict of interest 